\title{
Factors in Assessing Performance of Wide Area Communication Networks for Distributed Con- trol of Power Systems
}

\author{
Moustafa Chenine, Lars Nordström, Member, IEEE and Pontus Johnson, Member, IEEE
}

\begin{abstract}
This position paper presents research that is being started on the subject of modeling and evaluating performance and related system quality concerns in design of information and communication technology platforms used for power system operation and control. The focus of the project is on how the performance and other quality aspects of such networks interact with modern power control system technologies such as for instance Wide Area Monitoring and Control (WAMC) systems. Specifically the paper presents an evaluation and modeling approach based on a set of theoretically grounded factors important for assessing performance of wide area control systems. These assessment factors are used to define the modeling semantics necessary for enhanced models of the information and communication technology platforms. The factors are further represented in Influence Diagrams, enabling analysis of their inter-dependence with for instance Bayesian analysis. This approach has the potential of offering decision support for design of high performance information and communication technology platforms, while at the same time considering other system qualities. The paper presents an overview of the suggested approach, complemented with influence diagrams describing the inter-dependence of performance factors. The paper also provides an example of application of the approach to a simple control system architecture.
\end{abstract}

\section{Index Terms-}

Communication system performance, Distributed Control, Power System Communication, Power System Control, Wide area networks

\section{INTRODUCTION}

$\mathrm{T}$ HE later years of development within information and communication technologies (ICT) have enabled power system control to make greater use of new types of sensors, communication systems, and distributed computer networks. Traditionally centralized SCADA/EMS solutions with local protection systems are merging into distributed control systems utilizing wide area measurements. With this

Manuscript received April 15th, 2007. This work was supported in part by the ELEKTRA program and by EKC ${ }^{2}$ - The Swedish centre of Excellence in Electric Power Engineering.

M. Chenine, L. Nordstrom and P. Johnson are all with the department of Industrial Information \& Control Systems, School of Electrical Engineering, KTH - The Royal Institute of Technology, SE-10044 Stockholm, SWEDEN. (phone: +46-8-790 6831; e-mail : \{moustafac |larsn|pj101\}@ics.kth.se). development comes an increasing dependence on reliability, interoperability and performance of the ICT platform supporting this distributed control system. Included in the platform is the communication network, information exchange mechanisms, as well as the servers and nodes providing the computing platform.

This paper presents a project that has been started to investigate the performance factors affecting the design of such ICT platforms. Since the platforms include communication, information exchange as well as computing components, the project addresses a wide range of performance aspects. For some areas, e.g. performance analysis of TCP/IP based networks; there are well known and established techniques and simulation tools available. For others, such as performance assessment of software built using Service Oriented Architectures, the area of performance assessment is not as well understood. Additionally, methods that combine assessment of several of these aspects, including related fields such as interoperability, information security and reliability are an unexplored field. To address this challenge, the project will use modeling and evaluation concepts developed for analysis of enterprise architectures [1]. This allows the inclusion of theory from other fields such as systems interoperability into the analysis of performance. Such methods use extended influence diagrams (EID), to create theoretically grounded assessment frameworks and related modeling semantics. The EIDs and metamodels are then used to create models of the ICT platform that can be used to assess the system qualities of alternative solutions.

\section{A. Outline of the Paper}

The paper begins with a brief narrative of the current development within power system operation and control, from hierarchical SCADA/EMS architectures towards distributed control systems. The purpose of the narrative is to provide a background to the problem being studied. Thereafter in section III, established methods for performance analysis of communication and computing platforms are introduced and the applicability of these methods to the challenges posed by distributed control systems is briefly discussed. In section IV, the approach for performance modeling and evaluation which is the topic of this research project is presented together with examples of basic factors for perform- 
ance assessment identified. The factors are presented in the form of an influence diagram. The paper is concluded in section $\mathrm{V}$ with a discussion of the applicability of the approach, and the planned research activities.

\section{Distributed Control of Power Systems}

Power system monitoring and control has for decades been built in a centralized architecture, with a SCADA and Energy Management System (EMS) located in a control centre. In the control centre, operators have been provided with analog measurements and digital indications from the power system via the SCADA system. This has allowed them to monitor and control the power system on a near real-time basis [2].

With the advent of new communication and computing technologies, numerous visions for future distributed control systems for power system operation and control have been created, see for instance [3]. In these architectures, the functionality needed for control and protection of the power system can be located at any computing platform within the distributed control system. One strong drive towards distribution of functionality comes from the separation of the entities operating the power system caused by the re-regulation of the power industry. Additional drivers, also an effect of the re-regulation, are the need to operate the power system more efficiently and closer to stability margins to support market development and introduction of new energy sources. This in turn requires tighter real-time control of the power system not possible in the traditional hierarchical system architectures. A third, indirect driver, is the appearance of new technologies from other industries that enables the distribution of functionality, which in turn allows utilization of lower cost de-facto standards.

Enabling technologies for these future distributed control systems come mainly from the computer and communications industries. At the lower levels, protocols like Ethernet and SDH/SONET provide the physical and logical link layers. The UDP/TCP/IP suite of protocols provides the necessary networking and transport capabilities. Thanks to utilization of these industry standard protocols and technologies a wide array of technologies for building distributed control systems can be applied. A prominent example here is the utilization of autonomous systems as is described in [4]. One challenge that this development poses is the need for unified representation of information about the power system that must be available at all times for the software components [5]. The power industry has addressed this in standards for system integration, for example the IEC TC57 Common Information model [6]. Additionally, the data modeling and communication protocols specified in the IEC 61850 standard [7] provide an additional means of distribution of functionality and exchange of data. Use of these standards provides the needed interoperability, but at the risk of introducing additional data traffic leading to reduced performance.
In summary, it can be assumed that future distributed control systems will be built upon an ICT platform that is a blend of higher and lower level protocols and information exchange mechanisms. High performance of the platform is critical for the applicability of new power system control concepts such as Wide Area Monitoring and Control [8]. In turn, the performance of the ICT platform will be dependent on the reliability and interoperability characteristics implemented in its software and hardware components.

\section{Performance AnAlysis}

Generally, performance of software, computer, and communication systems is analyzed by measuring or predicting one or more characteristics of importance. These characteristics make up what is implied by performance. For example, these characteristics can be the response time of a system, a system's capacity, a communication link's bandwidth, a node's queue length or the degree of packet loss in a communication network. There are two basic categories of methods for conducting performance evaluation. These methods are (1) measurements of existing systems and measurements and (2) predictions of models that abstract existing or upcoming systems [9]. These models can then be further divided into analytical modeling and simulation modeling.

Measurements of existing systems are the most accurate techniques, since we can measure all aspects of interest without imposing abstractions or assumptions. The drawback of this method is that it is specific to the system being measured and it is difficult and sometimes impossible to measure variations in the performance characteristics as the systems parameters change [10]. Doing so would mean changing an existing system which could mean taking the system offline and out of operation.

Analytical modeling involves defining a model of the system that expresses the relation between the performance characteristics and other system parameters. Analytical modeling usually involves a number of assumptions on the system and its environment [10]. For example, for a node in a network, the sources of traffic could be assumed to be infinite and the arrival pattern assumed to be random and characterized as a Poisson distribution. This is usually not the case, specifically for example in industrial communication settings were traffic is either periodic or sporadic or both [11]. Two prominent techniques within analytical modeling are network calculus and queuing theory. Network calculus is originally targeted at communication network performance, specifically towards services guarantees and deterministic traffic [12]. Queuing theory on the other hand has been applied for both communication [13] and computer systems [10] where systems are modeled in terms of service times and queue lengths of nodes and traffic is represented in varying arrival patterns. Queuing theory is probability based and gives a statistical estimation of the parameter and characteristics of interest. 
The general drawback of analytical methods is that as the system gets more complex the methods also gets less feasible since the models of the system grow in complexity. To be manageable, analytical models focus on a limited set of parameters while ignoring the effect of other system parameters or properties which may also affect the performance of the system. This is especially true of factors that have an indirect effect on performance such as semantic interoperability or information security. It can be assumed that system design based merely on performance analysis may lead to a local optimum in terms of performance of the system, while risking lower level of interoperability or reliability [14].

Simulation involves mimicking the behavior of existing or planned systems and observing the change in performance based on changes in modeled system parameters. Generally speaking, the parameters can be more varied than those involved in analytical analysis. Example of a general purpose simulation package is OPNET [15] which can model many characteristics of communication networks. Especially targeting Power System Control, a set of simulators have been combined into a platform, EPOCH. In [16] the EPOCH simulator is used to model different protection and control schemes including power system as well as communication system characteristics. Yet simulation still can not model every single detail of the system and involves assumptions and simplifications in order to implement the program and to execute it in a reasonable amount of time [10].

\section{A. Applicability of analysis methods}

As described in section II performance of modern distributed control systems is dependant on the performance of the ICT platform's computing, information exchange and communication mechanisms. This includes aspects on performance not traditionally covered in performance analysis methods, for example interoperability or reliability. As exemplified in [16] it is perfectly feasible to create communication models and integrate these with Power System simulators. However, these models lack aspects on performance that have an effect on the real-world system, for example the performance implications of the semantic interoperability of applications. This is especially true if the modeling and evaluation effort is to be made at an early stage in the design process, where many system characteristics and environmental factors are unclear. At this stage, creating a model that can be simulated may involve as much time and resources as designing the system itself.

\section{SUGgESTED APPROACH}

The research project presented herein intends to address the two weaknesses indicated in the preceding section. First, we want to have an evaluation and modeling framework that allows us to model the ICT platform at varying levels of abstraction and incorporate appropriate level of detail depending on maturity the system design. Second, we wish to include the impact of performance related fields, such as interoperability, maintainability and reliability in the modeling and evaluation. The approach is of course based on utilizing existing performance assessment methods, like queuing theory and simulation where applicable. The added value of the modeling and evaluation approach lies in its ability to include theory from other system quality fields including heuristics and qualitative knowledge about design and behavior of software, communication systems and information exchange mechanisms.

\section{A. The Metamodel}

To assess performance of any system, we need a model of the system. A suitable starting point for such modeling is a metamodel that defines the rules and structure of modeling. We propose a basic metamodel that defines two classes, a link and a node and a relation between these see Figure 1. These super-classes can then be used to create more detailed models using traditional object-oriented inheritance principles. For example, for a specific type of node, for example an IED, a set of additional attributes may be necessary for a complete model. Such additional attributes and how they are added in inherited classes is described in section IV.C below.

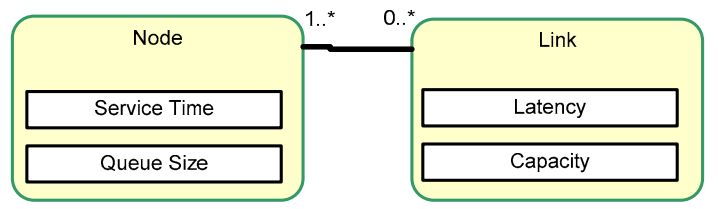

Figure 1. The basic metamodel, and its two super classes.

In the metamodel, Nodes have the attributes service time and queue size, the links have attributes latency and capacity. The meta-model allows a modeler to capture concept similar to those used in queuing theory. A basic queuing theory metamodel applied to asses a router would, for example, include such concepts as server i.e. the router itself, service time that it would take to serve the jobs which are basically requests or data. In the case of the router, jobs would be the frames arriving from the system's environment. In queuing theory it is assumed that jobs can be serviced one at a time, therefore a queue is formed, and the queue therefore is formed in the router's buffer. Systems can only hold limited amount of queues, therefore the queue length or size is another important characteristic[10].

In short, the nodes and links super-classes proposed here can represent instantiations of any software; computer or communication system on that level of abstraction, very similar to what is possible when using for instance queuing theory. Referring to the example system in Figure 2, the IEDs, Routers, and Control system servers could all be represented as Nodes having service times and queue sizes. While the LANs and the TCP/IP network could be represented by the Link classes with its constituent attributes. We could then utilize traditional queuing theory to analyze the performance of the system. However, to expand upon the capabilities provided by this analysis method, we sug- 
gest the inclusion of additional attributes to the classes. This would allow more sophisticated modeling of the entities involved in an ICT platform for wide area distributed control system.

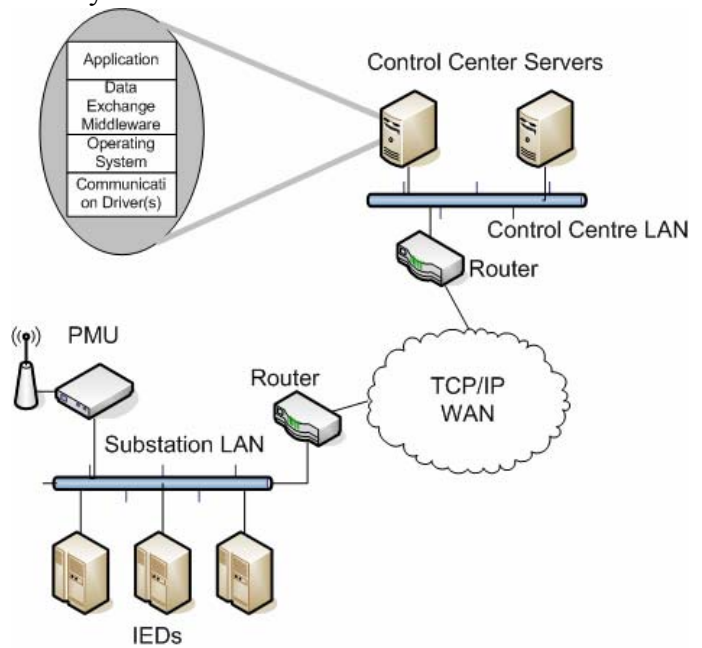

Figure 2. Example network, consisting of a substation LAN connected to the control centre through a TCP/IP based WAN.

\section{B. An Extended Metamodel}

To capture knowledge about software, computer and communication system design and behavior the addition of attributes to the basic top-level classes is necessary. There are a number of factors that affect the service time and queue size such as processor cache memory, speed of RAM memory, quality of the I/O Subsystem, execution and resource scheduling algorithms, prioritization mechanisms and so on. Addition of such attributes would give a wider and deeper assessment of the many factors and design issues need for a high performance system. Figure 3 illustrate some additions of attributes to the super-classes of the basic metamodel. The three new attributes added to the node are: software cohesiveness, capability of memory and efficiency of communication protocol.

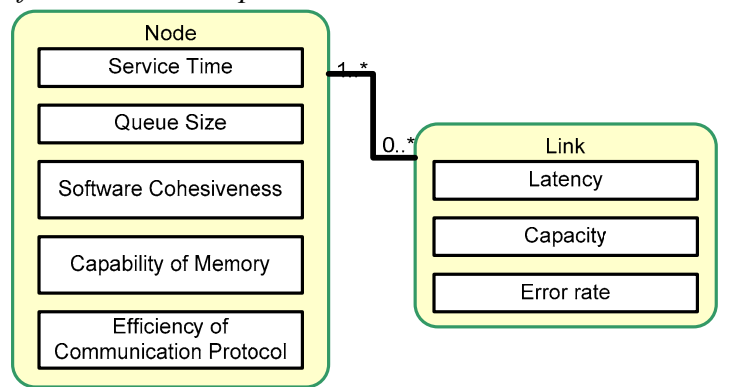

Figure 3. the expanded super-classes. The added attributes are merely examples. Which attributes should be added is dependant on what the model shall reflect.

Keeping a logically cohesive structure in a software system keeps overhead messaging to a minimum. This increases the service time, since the latency incurred from inter component coordination is also minimized. An example of a design with low cohesion is the use of a "god" class in object-oriented programs. A "god" class serves the purpose of keeping track of and initiating events in other classes, which in turn exhibits excessive messaging traffic due to the separation of functions and their related data [17]. The capability of memory is also another determinant of system performance. Random Access Memory (RAM) has a primary effect on performance, since it holds the bulk of the real time information waiting to be processed etc. for example, in the case of web based systems. The larger the available RAM the better the response time and throughput of the system [18].

Efficiency of the communication protocols also has a significant impact on a system. The communication protocol determines routing, congestion and error control, efficiency of these algorithms and the behavior of the protocol. For example TCP/IP exhibits two problematic features which may be problematic for power system communication Random Early Drop (RED) and TCP "Slow Start" [19]. RED is a router policy which attempts to predict possible overload on the network and starts to randomly discard packets even before the buffer is overloaded. TCP also varies the data rate so that receiving nodes are not overloaded, in TCP slow start, the throughput rises quickly and then falls again, if the connection become idle, TCP resets to low data rate. These features affect the service time since nodes have to wait for retransmission of discarded packets and introduce an unnecessary degree of latency.

The additional attribute added to the link is the error rate which is due to the inherent properties of the physical medium or due to the environment in which the link is placed. For example in substations or on power lines, these environments can cause electromagnetic interference which could distort the signal and thus the data sent through it [20].

To clarify the modeling concept we present the example depicted in Figure 2. The example illustrates an arbitrary control centre application based on phasor measurements. The specific application is not distributed in the sense discussed in section II above, but does still exhibit strict realtime requirements necessitating performance analysis. For purposes of the example, the system has been simplified and consists of a PMU connected to a LAN at a substation. The substation LAN is connected to the control centre via a TCP/IP based WAN. At the control centre, a server runs the application. This application is built on a stack of software consisting of communication driver, operating system and data exchange mechanisms, e.g. OLE for Process Control (OPC).

By creating a model of the example system based on the extended metamodel we can represent the different nodes with relevant attributes. For example, referring to Figure 2, we can model IED attributes such as queue size, memory capability and communication protocols. Furthermore, communication protocol has an impact since delay can be introduced due to decoding and protocol layering. In addition, all router and control center systems can be also repre- 
sented as nodes that are receiving jobs from the PMUs and IEDs for processing. In this case the cohesiveness of these systems in terms of software has an impact, since the introduction of delays due to cohesiveness would contribute to the degradation of performance. The communication network, i.e. the substation and control centre LAN and the TCP/IP WAN can also can be represented as links which have features such as latency, capacity and error rate depending on the operational environment in which they are installed.

\section{Relation between the added attributes}

It is of course important, that the attributes added to the modeling classes add actual value to the output of the analysis, and that their relation to existing attributes can be modeled and analyzed. Only attributes that have been derived from scientific knowledge and empirical studies can be added to classes in the meta-model. To enable analysis of the attributes, we propose that the casual relations between these attributes are represented in influence diagrams. An influence diagram is an extension of Bayesian networks that graphically represents causal relations between attributes, and each attribute in turn represents a variable with a number of states. Bayesian networks are also able to represent the uncertainty of causal relations using probabilistic reasoning. Figure 4 depicts the attributes added to the basic metamodel and illustrates the causal relations between them.

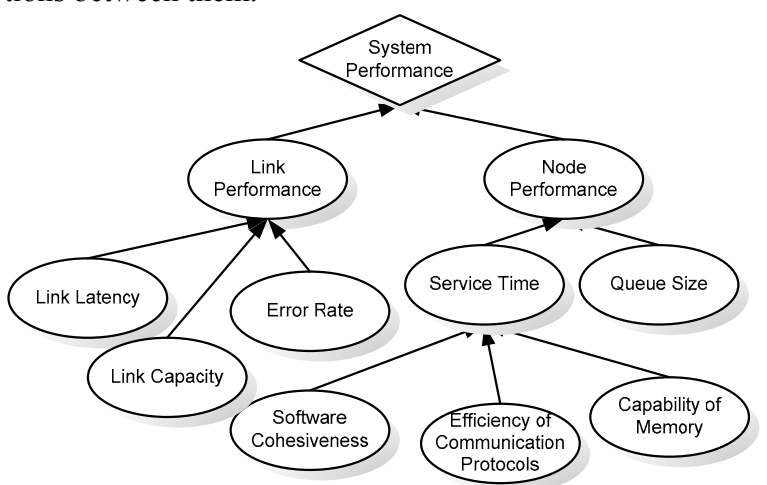

Figure 4. Influence diagram illustrating the causal relation between attributes

Three nodes appear in the influence diagram that are not depicted in the extended metamodel, these are System Performance, Node Performance, and Link Performance. The attributes in the extended model are factors that influence or determine the performance of the link and node, and therefore have a causal effect on the performance of that class. Finally, the system performance is an aggregation of the nodes and links that it is composed of. The additional attributes added in the previous sub-section have been illustrated in the diagram as influencing the service time, which in turn influences the node performance. As mentioned earlier these attributes decrease the service time if they are increased (in the case of software cohesiveness) and optimized (in the case of capability of memory and the effi- ciency of communication protocols). If we measure these for example and we find that the service time is sufficient, but the messaging traffic is high, then we can conclude that we can further decrease the service time by increasing the software's cohesiveness.

\section{Discussion}

By using inheritance to include additional attributes to the modeled objects and allowing the analysis of these attributes using Bayesian logic the proposed modeling and evaluation framework allows more sophisticated modeling of the ICT platform than is possible using traditional techniques. The level of abstraction that we want to use when evaluating a system, as well as the level of knowledge we have about the specific system may vary. Perhaps we want to break the system down to individual pieces of software in routers or applications, and therefore asses specifically the performance of the software system. We could also assess entire systems of systems ranging from details from hardware configurations and architectures to network configurations and architectures, and including for each specific node the application and operating system performance. The option of analysis at a higher level of abstraction is still possible and feasible. The method allows for a systemic performance analysis approach, i.e. analysis of system wide performance factors and their interdependence.

The above presentation should not be interpreted as if the proposed modeling and evaluation framework should be used instead of traditional analysis and simulation techniques. Rather it should be seen as an extension of these techniques that allow:

1. Inclusion of heuristic knowledge not properly represented in traditional performance analysis techniques.

2. Analysis of the indirect effect on performance imposed by for instance interoperability

3. Design support when attributes necessary for traditional analysis cannot be assessed or measured for instance during early design stages, when much of the system is unknown.

For instance, some values of the attributes in the influence diagrams can either be measured, or derived through other methods like simulation and queuing theory. However, since some of these values may not be readily available, the representation of the attributes and their relation in Influence Diagrams allows us also to cater to cases of uncertainty. There is a number of relevant and related research initiatives on the topic of using Bayesian logic for performance analysis, for example, in classification of traffic characteristics [21] and in identifying lossy links [22]. Bayesian networks are also applied in commercial simulation packages most notably in OPNET's Panorama Performance Analysis Engine [23]. By use of influence diagrams, interdependence between factors, including those not directly related to system performance can be included in evaluation. This is possible since the influence diagrams describing the attributes to be included in the extended classes can 
be chosen so that they represent attributes from another field of analysis such as reliability or interoperability. Figure 5 illustrates how some attributes that influence system performance also influence system reliability and interoperability. For a more complete discussion on the subject please see [1] and [24].

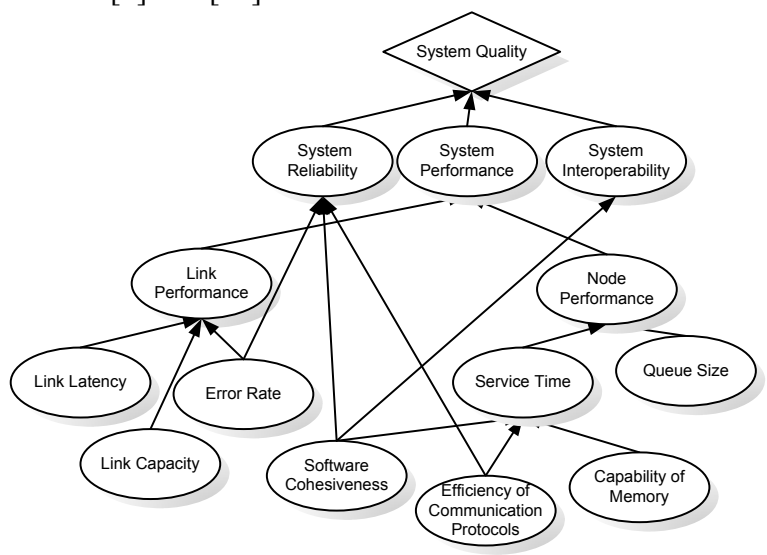

Figure 5. Influence diagram illustrating causal relation between attributes and to system reliability, performance and interoperability.

\section{CONCLUDING REMARKS}

As stated, this is a position paper describing the outlines of a research project. The approach presented herein, is intended to provide a complement to traditional, analysis and simulation based approaches to assessment of performance aspects of information and communication technologies. At this stage in the project, performance related factors from both communication as well as software have been compiled and described in influence diagrams. These diagrams have further been integrated with similar diagrams describing other areas of analysis such as interoperability, reliability and information security.

Concurrently, modeling semantics, as presented in this paper, have been developed and first attempts at modeling relevant real world systems are being made. The purpose of these models is to highlight areas where simulation techniques could be sufficient to determine some first order approximations of relevant attributes. Additionally, studies are underway in which traffic patterns representative of wide area control systems will be modeled.

\section{REFERENCES}

[1] P. Johnson, R Lagerström, P. Närman, and M. Simonsson, Enterprise Architecture Analysis with Extended Influence Diagrams. To appear in Information System Frontiers, 2007.

[2] T. Cegrell Power System Control Technology, Prentice-Hall, 1986

[3] F. Wu, K. Moslehi, A. Bose, "Power System Control Centers: Past, Present and Future" In Proceedings of the IEEE, Vol. 93, No. 11, November 2005.

[4] C. Rehtanz (Ed.), Autonomous Systems and Intelligent Agents in Power System Control and Operation Springer Verlag, Berlin-Heidelberg 2003, pp 1-37.

[5] Kostic, T, Kreiss, O., "Unified Information Models in Support of Location transparency for Future Utility Applications" in Proceedings of the 39th Hawaii International Conference on System Sciences.
[6] IEC, International Electrotechnical Commission, "IEC 61970-301 Energy management system application program interface Part 301: Common Information Model (CIM) Base", IEC Reference number IEC 61970-301:2003(E).

[7] IEC, International Electrotechnical Commission, "IEC 61850-7-4 Communication networks and systems in substations - Part 7-4: Basic communication structure for substation and feeder equipment Compatible logical node classes and data classes", IEC, Reference number IEC 61850-7-4:2003(E).

[8] M. Zima, M. Larsson, P. Korba, C. Rehtanz, G. Andersson; "Design Aspects for Wide- Area Monitoring and Control Systems"; Proceedings of the IEEE, Vol. 93, No. 5, May 2005, pp .980-996

[9] P. Fortier, H. Michel, Computer Systems Performance Evaluation and Prediction, Digital Press, 2003.

[10] D. Lilja, Measuring Computer Performance: A Practitioners Guide, Cambridge University Press, 2000.

[11] J.D. Decotignie, Ethernet-Based Real-time and Industrial Communications, Proceedings of the IEEE, Vol. 93, No. 6, June 2005.

[12] J. Le Boudec, P. Thiran, Network Calculus, A Theory for Deterministic Queuing Systems for the Internet. Springer-Verlag Berlin/ Heidelberg, 2001.

[13] G. N. Higginbottom, Performance Evaluation of Communication Networks, Artech House Publishers, 1998.

[14] M. Barbacci, M.H. Klein, T.A. Longstaff, C.B., Weinstock., Quality Attributes, Technical Report CMU/SEI-95-TR-021, December 1995

[15] OPNET Modeler [Online]. Available at http://www.opnet.com.

[16] K. Hopkinson, X Wang, R. Giovanini, J.Thorp, K.Birman, D. Coury, EPOCHS: A Platform for Agent-Based Electric Power and Communication Simulation Build From Commercial Off-the-shelf Components. IEEE Transactions on Power Systems.Vol. 21, Issue 2, May 2006.

[17] C. U. Smith, L. G. Williams, Performance Solutions: A Practical Guide to Creating Responsive, Scalable Software. Addison-Wesley Professional, 2002.

[18] M. Andreolini, M. Colajanni, R. Lancelloti, Impact of memory technology on the performance of Web Systems. Proceedings of the International Conference on Next Generation Services Practices, IEEE Computer, 2005.

[19] K. P. Birman, J. Chen, E. M. Hopkinson, R. J. Thomas, J. S. Thorp, R. Van Renesse, W. Vogels, Overcoming Communications Challenges in Software for Monitoring and Controlling Power Systems, Proceedings of the IEEE, Vol. 93, No.5, May 2005.

[20] D. J. Marihart, Communications Technology Guidelines for EMS/SCADA Systems. IEEE Transactions on Power Delivery, Vol. 16, No.2, April 2001.

[21] A. W. Moore, D. Zuev, Internet Traffic Classification Using Bayesian Analysis Techniques. ACM SIGMETRICS Performance Evaluation Review, Vol. 33, Issue 1, June 2005.

[22] V. N. Padmanabhan, L. Qiu, H. J. Wang, Passive Network Tomography Using Bayesian Inference. Internet Measurement Workshop 2002 (IMW 2002).

[23] Understanding Panorama's Performance Analysis Engines, OPNET Whitepaper, OPNET Technologies, Inc, 2005

[24] P. Johnson, R. Lagerström, P. Närman, M. Simonsson, System Quality Analysis with Extended Influence Diagrams. In Proceedings of the System Quality and Maintainability Workshop (SQM '07). 